\title{
Current Pattern Analysis for Oil-Spills - A Case Study Using San Francisco Bay
}

\author{
Jerry A. Galt \\ Hazardous Materials Response and Assessment Division \\ Nation Ocean Service, NOAA \\ Seattle, WA , USA \\ Ralph T. Cheng \\ Water Resources Division \\ U. S. Geological Survey \\ Menlo Park, CA, USA \\ Wayne L. Wilmot \\ National Ocean Service, NOAA \\ SilverSpring, MD , USA
}

\begin{abstract}
During oil-spill events the movement and spreading of the pollutant is of critical interest to responders and there are always demands for forecasts of the future position of the oil. For floating portions of the spilled oil that are constrained to remain on the surface, the two-dimensional kinematics of the flow patterns can be used to identify locations for flotsam collection. In areas where there are strong "flow convergencies" a Lagrangian view shows the surface water moving towards a common region. As a result, the concentrations of floating material will increase where convergence zones are found. In coastal communities these areas are typically referred to as "tidal rips" or "rips". In some regions these convergence zones are persistent enough to have common names associated with them. The convergence zones are always areas for flotsam collection.

A high-resolution now-casting numerical model has been implemented for San Francisco Bay to assist oil-spill prevention and response. Thirty-six hours of now-casting highresolution numerical model results are available centered around the present time. The current patterns from these model results can be further analyzed to provide information that identifies areas of strong convergence. Results of this kinematic analysis can be used to identify likely collection areas for floating oil and to provide location maps for the effective placement of recovery equipment. Convergence zones are typically associated with barotropic flow over large bathymetry variations or density fronts at interfaces where oceanic and fresh water mix. These analysis techniques were further developed and tested in San Francisco Bay to strengthen local oil-spill response planning activities. During an actual spill caused by the Cape Mohican in San Francisco Bay (late October 1996), the planning response for clean up efforts and the development of insights on the movement of oil patches were enhanced by the results of the current pattern analysis.
\end{abstract}

\section{Introduction}

Describing the advection and diffusion of a pollutant in a fluid environment is a common enough problem that comes up in oceanography, atmospheric sciences, and civil and environmental engineering. Most flows of geophysical interest can be considered as incompressible and this has a profound effect on the type of solutions that are possible with the standard mass balance equation. For a conservative pollutant, this 
can be written:

$$
\frac{\partial C}{\partial t}+\nabla \cdot(\vec{V} C)=0
$$

Expanding the divergence of the mass flux, which is represented by the second term on the left side, gives:

$$
\frac{\partial C}{\partial t}+(\vec{V} \cdot \nabla) C+C(\nabla \cdot \vec{V})=0
$$

For a three-dimensional and incompressible flow the last term on the left side is identically zero, but for a pollutant that is confined to a two-dimensional surface, the above equation can be rewritten as:

$$
\frac{\partial C}{\partial t}+\left(\vec{V}_{h} \cdot \nabla\right) C=-C\left(\nabla \cdot \vec{V}_{h}\right)
$$

Only the components of the flow along this surface affect the distribution of the pollutant. It is hard to over emphasize the significance of this difference (Galt 1994).

Consider, for example, pollutant in the form of smoke leaving a smoke stack. The smoke moves through a three-dimensional space and continues to decrease in concentration along its trajectory. This is a consequence of the parabolic form of the mass balance equation with no source term, and it is impossible for the smoke particles to ever recoalesce because of simple advection and diffusion processes. (Obviously rainout and chemical interactions are not represented by this equation.) The same condition is not even approximately true for floating oil. The term on the right side of the last equation acts as a source term and concentrations can increase along a trajectory. As oil ages and spreads out, it typically breaks into small patches referred to as "tarballs." These may spread out over very large areas and become impossible to detect visually. As these scattered tarballs drift into a two-dimensional surface convergence (i.e., a region where vertical movement carries water away from the surface) they will recoalesce and apparently recreate the spill. This has happened numerous time, even at trajectory distances of several hundred kilometers. In reality, the final distribution of all large oil spills and any response activities aimed at their mitigation are controlled by the convergence patterns in the path of the spill. The authors know of no pollutant recovery system that works in a gas or liquid where the pollutant is subject to unconfined, three-dimensional spreading. No one ever tries to recover any component of smoke after it has left a stack and oil spill recovery equipment has no practical options for recovery unless it is associated with a natural convergence zone, or the development of a synthetic one like a beach or boom.

Strong convergences in natural geophysical domains are associated with one of two physical processes. The first is associated with barotropic flow from a shallow region to deeper water. An example can be found in Lower Cook Island where a feature known locally as the "main rip" is found east of Kalgin Island. This captured and held virtually all of the oil released in the spill from the Glacier Bay which took place near Kenai Alaska. The second physical process that causes strong convergencies is associated with the formation of a fresh water lens that floats over more dense salt water. These are obviously associated with a fresh water source and perhaps the most conspicuous oil-spillrelated example came from the LouisianaTexas coast where the Mississippi Atchafalaya runoff forms a narrow band of fresh water along the coast. The oil from the Alvenus spill spread along 125 miles of this front but the slick remained only a few meters wide. For both of these cases velocity components normal to the lines of convergence were measured to be in excess of $10 \mathrm{~cm} / \mathrm{sec}$.

With this in mind, it is obvious that an understanding of regional convergence zones 
and their relationship to the pollutant trajectories is essential to accurately describing the movement and spreading of oil spills.

\section{Model Results}

For estuarine numerical models to resolve naturally occurring convergence zones requires a great deal of computational power. Barotropically controlled convergences require high resolution of the bathymetry and dense computational grids. Baroclinically controlled convergence zones require the same high degree of spatial resolution and the inclusion of highly nonlinear terms in the equations that represent the processes that are important for frontogenesis. Although these types of models exist in research settings there are very few that are designed to be used operationally. One such model has been developed for San Francisco Bay by Cheng et al. (1993). This model has a spatial resolution of 250 meters (Cheng et al. 1997) and does a good job of resolving the bathymetriclly controlled convergence zones that are induced by the tidal flow. A highresolution baroclinic version of the model is also under development.

It would be useful to understand how strong a convergence zone would have to be to force the floating pollutant to recoalesce and overcome the effects of turbulent diffusion, or spreading. From dimensional analysis considerations A non-dimensional term can be found that represents the effects of advective convergence divided by the effects of an effective dispersion coefficient.

$$
\frac{\left(\nabla \cdot \vec{V}_{h}\right) L^{2}}{K}
$$

If this term is greater than one, convergence factors will tend to dominate the spreading of the floating pollutant. A series of numerical experiments carried out on a simple advection/diffusion model representing the mass balance given in equation (3) confirmed these results. An analysis of the current patterns generated by the San Francisco model indicated that there are a number of banded convergence zones that appear capable of trapping a scattered floating pollutant. These areas are all associated with the edges of channels where the ebb tide sweeps water from the surrounding flats into the deeper waters of the channels. The elongation of the water column causes a convergence of the surface area which collects the pollutant.

\section{Application to spill Response}

In Fall 1996 near the end of October, an oil spill occurred in San Francisco which provided an opportunity to test a number of the ideas that were outlined in this note. The ship Cape Mohican which was in dry dock along the San Francisco waterfront fell victim to a plumbing error that released a significant quantity of bunker oil into the Bay. An immediate response was initiated under the standard regional response plan developed for the San Francisco Bay area. Under this plan the United States Coast Guard has the Federal government's lead as part of the unified command. NOAA's Hazardous Materials Responseand Assessment Division provides technical support to the Coast Guard and Unified Command including trajectory analysis. As a result of the detailed current pattern analysis that had been under investigation as part of the authors joint research project the trajectory analysis team knew the detail that would be required in the supporting hydrodynamic models that were used for the trajectory analysis. The standard models were modified to include the necessary high resolution. A test analysis of the patterns confirmed that the response models were able to resolve the major convergence patterns that were found in the detailed model developed by Cheng et al. (1997). The significance of the convergence patterns in San Francisco Bay can easily be 
seen in Figures 1 and 2, which were taken from the results of the trajectory support model that was used in real time for operational support during the spill. The first figure indicates the distribution of floating oil on 10/30/96-1400. The second figure shows the trajectory analysis results six hours later and clearly indicates the result of strong convergences collecting the oil into a large banded structure south of Angle Island that leads off towards the Golden Gate. These modeled oil distributions and the actual convergence zones observed during careful overflights showed useful correlations. The results were then used to help position recovery equipment and represented a new level of technical support for real spill operations.

\section{Conclusions}

The results of the current pattern analysis carried out for this project indicate that high-resolution estuarine models can resolve important kinematic flow features that are essential to understanding the movement and spreading of floating pollutants. That this high level of technical support is possible suggests that models that provide less than this will not be acceptable in responding to future spills. The next level of improvement can be expected when the research models can be operationally configured to support real time emergency response. This is primarily a matter of software engineering and is under consideration in continuing joint work by the authors.

\section{References}

Cheng, Ralph T, Vincenzo Casulli, and Jeffrey W. Gartner. 1993. Tidal, Residual, Intertidal Mudflat (TRIM) model and its applications to San Francisco Bay, California. Estuarine, Coastal and Shelf Science (36):235-280.

Cheng, Ralph T, Wayne L. Wilmot, Jerry A. Galt 1997 A Nowcasting Model for Hazard
Spill Prevention and Response, San Francisco Bay, California. Proceedings Ocean'97 MTS/IEEE (in press).

Galt, J. A. 1994. Trajectory analysis for oil spills J. Adv. Mar.Tech. Conf. (11):91-126. 
Figures

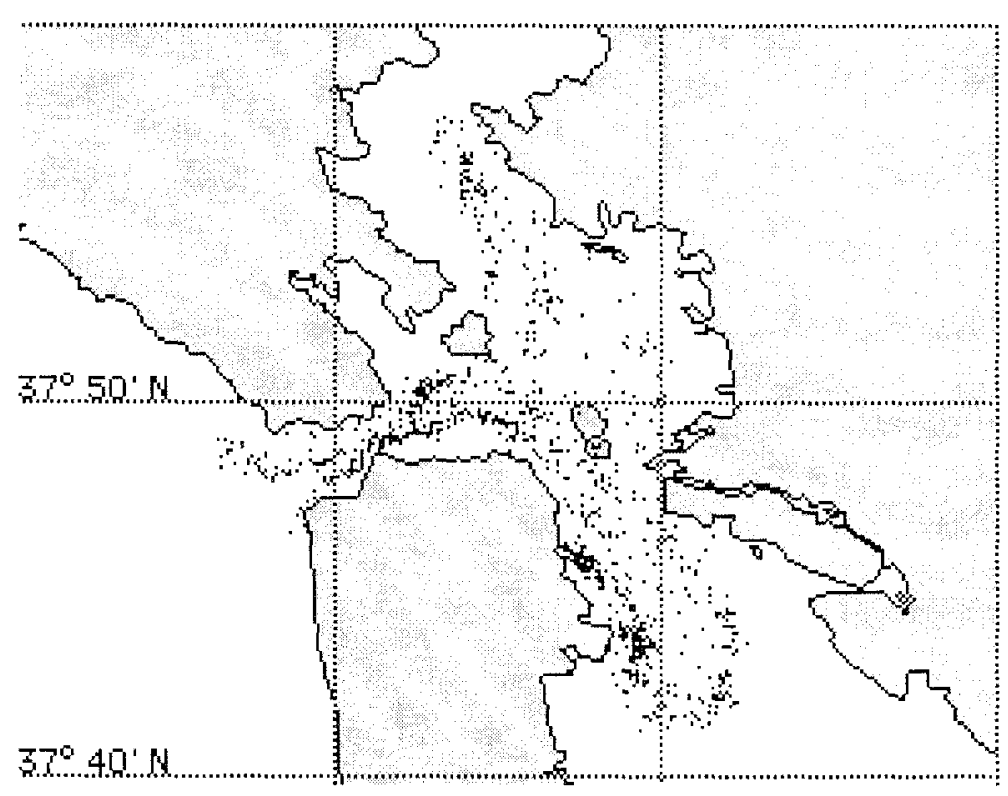

Figure 1 - Predicted oil distribution at Oct. 30, $19961400 \mathrm{hr}$ Distribution is seen to be widely scattered

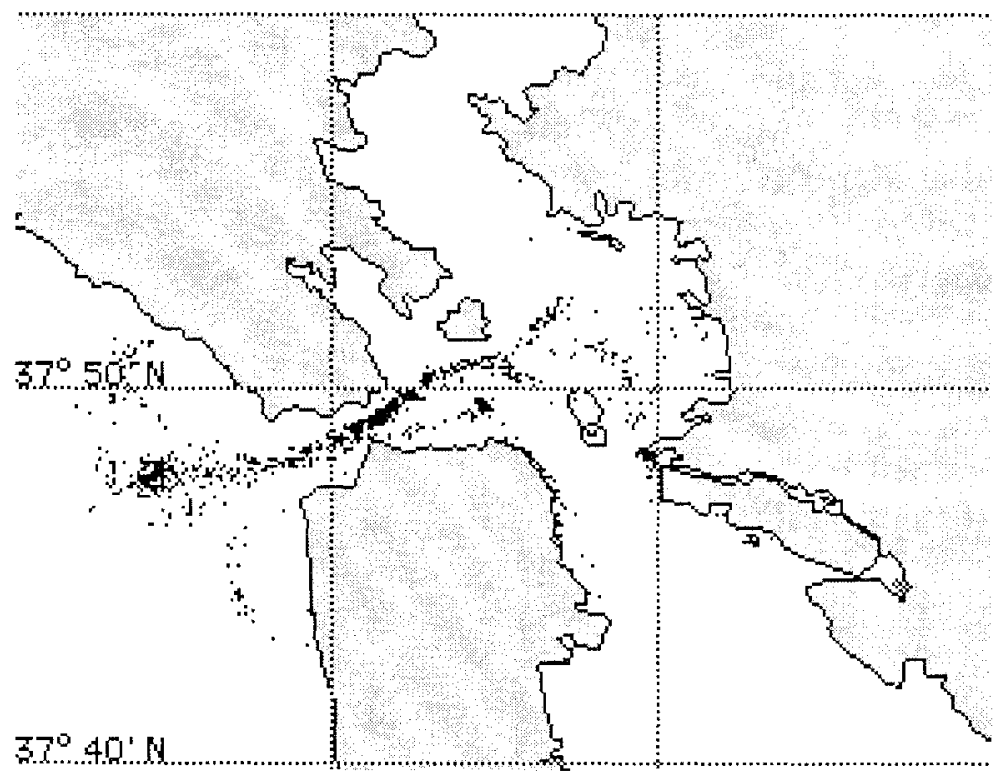

Figure 2 - Predicted oil distribution at Oct. 30 , $19962000 \mathrm{hr}$. The oil distribution is seen to be collected in well defined convergence zones. 